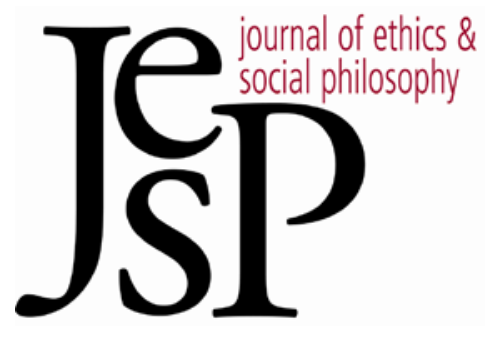

\title{
Is $\mathrm{I}_{\mathrm{T}} \mathrm{B}_{\mathrm{AD}} \mathrm{T}_{\mathrm{O}} \mathrm{B}_{\mathrm{E}} \mathrm{D}_{\text {ISABLED? }}$ \\ Adjudicating Between the Mere-Difference and the \\ B AD-Difference $_{\text {IEws of }}$ Disability $_{\text {If }}$
}

BY VUKO ANDRIĆ AND JOACHIM WÜNDISCH

JOURNAL OF ETHICS \&SOCIAL PHILOSOPHY

Vol. 9, No. 3| DECEMBER 2015 URL: WWW.JESP.ORG 


\section{Is It Bad to Be Disabled? \\ Adjudicating Between the Mere-Difference and the Bad-Difference Views of Disability}

Vuko Andrić and Joachim Wündisch

\section{I} N HER RECENT ARTICLE, "VALUING DISABILITY, Causing Disability" Elizabeth Barnes has defended the mere-difference view of disability. ${ }^{1}$ According to this view, disability does not by itself make disabled people worse off on balance. Rather, if disability has a negative impact on wellbeing overall, this is only so because society is not treating disabled people the way it ought to treat them. In objection to the mere-difference view, it has been argued, roughly, that the view licenses the permissibility of causing disability and the impermissibility of causing nondisability. ${ }^{2}$ Barnes attempts to show that this causation-based objection does not succeed. We disagree and argue why. While Barnes' specific argument is unconvincing, it unearths an important challenge for anyone who wishes to adjudicate between the mere-difference and the bad-difference views of disability in a way that enables a dialogue between the proponents of both views. ${ }^{3}$ We offer considerations to overcome that challenge.

The paper proceeds as follows. In sections I and II, we present the causation-based objection to the mere-difference view. In section III, we critique one defense strategy against it. In section IV, we discuss a second, much more powerful strategy to this effect. In section $\mathrm{V}$, we show the limits of this strategy. Section VI is our conclusion.

\footnotetext{
${ }^{1}$ E. Barnes (2014) "Valuing Disability, Causing Disability," Ethics 125: 88-113.

2 For variants of this objection see the articles mentioned in Barnes (2014: 93, n. 6); J. McMahan (2005) "Causing Disabled People to Exist and Causing People to Be Disabled," Ethics 116: 77-99; J. Harris (2001) "One Principle and Three Fallacies of Disability Studies," Journal of Medical Ethics 27: 385-87; G. Kahane (2009) "Non-Identity, SelfDefeat, and Attitudes to Future Children," Philosophical Studies 145: 193-214; P. Singer (2001) "Ethics and Disability: A Response to Koch," Journal of Disability Policy Studies 16: $130-33$.

3 The causation-based objection aims at establishing such a dialogue but can only achieve it between proponents of the mere-difference view who have not fully grasped the implications of their view and proponents of the bad-difference view. Most troubling is the challenge of creating a dialogue with proponents of the mere-difference view who have accepted all implications of their view. G. Kahane and J. Savulescu ((forthcoming) "Disability and Mere Difference," Ethics) forgo that challenge and do not attempt to convince those committed to the intuitions that support the mere-difference view. A further and related difference between our critique of Barnes' article and that of Kahane and Savulescu is that we directly criticize the mere-difference view and not merely Barnes' claim that the mere-difference view does not have unacceptable implications.
} 


\section{The Causation-Based Objection and the Defeat of Strict Infer- ence}

Barnes observes that the bad-difference view - according to which disability makes people worse off by itself - is normally taken to be the commonsense view of disability by philosophers. As a proponent of the mere-difference view, Barnes is concerned with the following counterargument: "If disability were mere-difference rather than bad-difference, it would be permissible to cause disability; it is obviously impermissible to cause disability; therefore, disability is not mere-difference; it is baddifference." 4 As proponents of this argument, Barnes mentions Jeff McMahan, John Harris, Guy Kahane and Peter Singer. ${ }^{5}$ It is clear that Barnes seeks to show that this argument fails. However, it is not perfectly clear - to us, at any rate - which part of the argument she seeks to challenge and on what basis she seeks to do so.

First, Barnes defends the claim that even if disability is meredifference it is not in all cases permissible to cause disability. In fact, she says outright:

[T] he objection I am opposing takes the form of a conditional: if disability is mere-difference, then it is permissible to cause disability (and likewise impermissible to remove or prevent disability). I am arguing that this inference is mistaken: it is not the case that if disability is mere-difference we can thereby infer that it is permissible to cause disability. ${ }^{6}$

To make this case, Barnes argues at some length and based on multiple examples that there are cases in which it is not permissible to cause disability even if disability is mere-difference. In one such case, one coworker blinds another while carelessly playing with a laser after hours. 7 According to Barnes, the defender of the mere-difference view can condemn this case of causing disability on a number of grounds. Consider two examples: First, becoming disabled involves significant transaction costs that arise through adjusting to the disability. Second, we should not interfere in the life of another person without justification, even if that interference is neutral with respect to wellbeing.

Barnes is certainly right in her assessment of this specific case and in her defense of the general claim that the mere-difference view does not license causing disability in all cases. Nevertheless, her approach raises questions. For one, properly interpreted, the objection to the meredifference view Barnes seeks to challenge does not rely on the claim that the inference from "disability is mere-difference" to "causing disability is permissible" works in all cases. Rather the objection must identify at least one class of such cases and show that this class of cases undermines the

\footnotetext{
4 Barnes (2014: 93).

5 Ibid.

${ }^{6}$ Ibid.: 94 . Similar references to this very limited claim are found in the abstract of the paper and on 88 .

7 Ibid.: 95.
} 
plausibility of the mere-difference view. Therefore, Barnes has at this stage supplied only a prelude to a potential counterargument.

More problematically, Barnes seems to imply that the authors she cites would actually support the strict-inference claim she has proven to be implausible. We find this textually unfounded ${ }^{8}$ and a rather uncharitable interpretation of the causation-based objection. For not only is the strict-inference claim unnecessary for the success of the objection but it is also clearly false as can be demonstrated based on any number of counterexamples (e.g., the person who expressly pleads not to be made disabled).

\section{Where the Views Diverge: Disabled Baby vs. Reverse Disabled Baby}

In order to defeat the causation-based objection, it does not suffice to show that it is not always the case that the mere-difference view licenses causing disability. Rather, license in some cases, in a way that undermines the plausibility of the mere-difference view, would suffice for the causation-based objection to succeed. Despite her in-depth treatment of the strict-inference claim, Barnes admits this. Therefore, later on in her paper, she rightly shifts focus to demonstrate that "mere-difference views of disability do not license the permissibility of causing disability ... in any way that undermines the tenability of the mere-difference position."' In short, she seeks to show that the implications of the mere-difference view do not discredit it. Barnes attempts to defend this assessment even though she accepts that the implications of the mere-difference view stand in contrast to the implications of the bad-difference view, which as mentioned above - she takes to be the commonsense view of disability among philosophers. Consider the following scenarios:

\footnotetext{
Disabled Baby

Cara has a six-month-old baby, Daisy. Cara values disability, and thinks that disability is an important part of human diversity. Moreover, she thinks that increasing the number of happy, well-adjusted, well-educated disabled people is an important part of combating ableism (and has a justified belief that any child she raises has a good chance of ending up happy, well-adjusted, and welleducated). With all this in mind Cara has Daisy undergo an innovative new pro-disability procedure. Daisy doesn't endure any pain from this, and she won't remember it. But, as a result, Daisy will be disabled for the rest of her life. ${ }^{10}$
}

\footnotetext{
8 McMahan (2005: 95-96) explicitly treats a number of reasons why the strict-inference claim is not justified. These are the same reasons Barnes relies upon. The other authors mentioned by Barnes do not appear to support the strict-inference claim either - see Harris (2001: 384); Kahane (2009, \10); Singer (2001: 133).

9 Barnes (2014: 113).

10 Ibid.: 97. Note that in the case of Disabled Baby, Cara is taken to have a "justified belief that any child she raises has a good chance of ending up happy." To some extent this description is question-begging as it will likely lead the reader to (subconsciously) presuppose the truth of the mere-difference view - at least in the case of Disabled Baby. Of course, given particular assumptions about the epistemic state of Cara, it is possible
} 


\section{Reverse Disabled Baby}

Cara has a six-month-old baby, Daisy, who is disabled. Cara values Daisy's happiness and well-being. Moreover, she thinks that Daisy will have a better chance of being happy, well-adjusted, and well-educated if she is nondisabled. With all this in mind Cara puts Daisy through a radical new treatment for infant disability. Daisy doesn't endure much pain from this, and she won't remember it. But as a result, Daisy will grow up nondisabled. ${ }^{11}$

Barnes mentions that most people will think that Cara acts wrongly in Disabled Baby. However, Barnes notes that the advocate of the meredifference view of disability can accommodate that judgment with the help of noninterference principles. According to these principles, we should "refrain from drastically altering a child's physical development." 12 Let us for the sake of argument accept that Barnes is right in her assessment that the advocate of the mere-difference view can vindicate the commonsense view of Disabled Baby.

The challenge Barnes needs to meet arises at a different junction: According to common sense, the actions of Cara in both cases are not on a par. In contrast to the commonsense assessment of Disabled Baby, most people believe that Cara does something good in Reverse Disabled Baby. Barnes recognizes that this divergence between the assessments of the two cases spells trouble for the defender of the mere-difference view and proposes two lines of defense. First, the mere-difference advocate could accept the discrepancy but insist that the mere-difference position can accommodate it. Second, she could deny the discrepancy and argue against competing intuitions.

\section{Disabled Baby and the Argument from Risk Differentials}

According to Barnes, it is important to develop the first line of defense although she believes that it ultimately fails - in order to demonstrate that a commitment to the mere-difference view does not require a particular position "on the cause/remove discrepancy." 13 It is unclear to us why Barnes believes that she can rely upon a position that she evaluates to be unsound. It would seem that, if the first line of defense and similar advances fail, then salvage of the mere-difference view does indeed require denial of the discrepancy, even though denying the discrepancy would of course be highly counterintuitive from the perspective of common sense.

Nevertheless, Barnes argues that in order to defend the evaluative discrepancy between Disabled Baby and Reverse Disabled Baby the advocate of the mere-difference view could explore the possibility of rea-

for her belief to be justified yet false. However, talk of justified belief is likely to lead the reader to assume that what is at issue is justified true belief. Also note that in the case of Reverse Disabled Baby, Cara is described as "thinking" rather than having a "justified belief” regarding the likely future of her child.

${ }^{11}$ Ibid.: 99.

12 Ibid.: 100.

13 Ibid. 
soning that Cara acts wrongly in Disabled Baby because "[c]ausing Daisy to be disabled is riskier than causing Daisy to be nondisabled." 14 According to Barnes this is well-founded because "[s]ight is much less likely to make Daisy unhappy than blindness." 15 In a footnote, Barnes is careful to point out that this position does not imply that being sighted is to be preferred to being blind - which would, of course, be at odds with the meredifference view. Rather, "[t]he greater risk to well-being associated with blindness could be largely or entirely due to how we treat blind people." 16

However, this argumentative strategy based on potential risk is deeply problematic. The defender of this strategy faces a dilemma: Is the greater risk to wellbeing associated with disability in Disabled Baby and Reverse Disabled Baby due to social injustice against disabled people? An affirmative answer would render the cases irrelevant because the meredifference view makes a claim about the connection of disability and wellbeing in the absence of social injustice. If the answer is negative, then the mere-difference view cannot accommodate the evaluative discrepancy.

The risks in Disabled Baby and Reverse Disabled Baby can be easily explained if they result from social injustice against disabled people. In an ableist world, it is likely that additional risks are associated with having a disability. Moreover, if these risks are sufficiently severe this insight can explain the evaluative discrepancy between Disabled Baby and Reverse Disabled Baby. However, this provides the defender of the meredifference view with little of value. In the introduction, we said that the defender of the causation-based objection can happily admit that the mere-difference view does not license causing disability in all cases. To see whether the objection goes through we have to examine whether a class of cases undermines the plausibility of the mere-difference view. This can only be cases in which the proponent of the mere-difference view cannot appeal to indirect reasons for sharing the verdicts of the baddifference view. Therefore, proponents of the mere-difference view must be able to maintain their general conclusions even - and especially - if

14 Ibid. Of course, the defender of the mere-difference view might want to appeal to other differences between Disabled Baby and Reverse Disabled Baby in order to explain the discrepancy. In Reverse Disabled Baby, Cara's central and laudable motivation is to benefit her baby, whereas, in Disabled Baby, Cara might even be accused of using her baby as a mere means to promote a disability culture or Cara's own welfare. Therefore, while Cara is directly concerned with increasing the expected wellbeing of her baby in Reverse Disabled Baby, the baby's expected wellbeing only functions as a constraint in the case of Disabled Baby. We are confident that the cases could be adapted so as to take into account this difference (e.g., if Cara lived in a flourishing disability community and acted in order to benefit her baby). However, even if we stick to the original description of the cases, the proposed solution will not get the mere-difference view of the hook because it can only account for part of the moral difference that seems to be present in these cases. This can be seen by comparing our intuitive reactions to Disabled Baby and Reverse Disabled Baby with how we react to situations in which a mother can rightfully be accused of using her baby as a mere means by treating the baby in a way that does not seem harmful.

15 Ibid.

16 Ibid. 
the relevant thought experiments do not entail risks based on social injustice that give them indirect reasons not to cause disability.

What is necessary to assess the causation-based objection, then, is framing the thought experiments in a way that avoids, as far as possible, giving the defender of the mere-difference view a basis for mimicking the implications of the bad-difference view. If we eliminate risk based on injustice against disabled people, however, the postulated risk differential based on society's unjustified treatment of disabled people would disappear. The defender of the mere-difference view hence has to claim that the greater risks to wellbeing are not due to social injustice. If this were true it would indeed provide the defender with a significant explanatory tool that could function in the context of thought experiments in a nonableist setting. However, this tool would come at the cost of a new challenge - namely, explaining how the greater risks to wellbeing of being disabled can be squared with the overall neutral effects of being disabled on wellbeing.

One feasible explanation would be that the variance of wellbeing among disabled people is greater than that among nondisabled people but that on average wellbeing is the same across these groups. While it is not impossible that this explanation is true, it is woefully ad hoc. Simply put, it is unclear why the cases of above average wellbeing should cancel out the cases of below average wellbeing among people with disabilities. A number of theoretical scenarios could help explain how these cases could cancel each other out in their effect on wellbeing among the group of disabled people. However, what the proponent of the mere-difference view needs is a realistic explanation for why, e.g., there would be relatively more cases of above average wellbeing among disabled people as compared to nondisabled people. Such an explanation is not forthcoming and would carry an even higher argumentative burden than the meredifference view, which is rather unassuming in comparison.

Another explanation of the greater risks associated with disabilities open to the proponent of the mere-difference view would be to claim that society is justified in treating people with disabilities in such a way as to add extra risks to being disabled. But this explanation is not very convincing either. First, there do not seem to be good reasons for thinking that the claim is true. Second, the claim, if true, would create further challenges for the mere-difference view because defenders of the view would have to explain why disability can be neutral for wellbeing in a just society even though that society exposes people with disabilities to higher risks than people without disabilities.

To sum up, accepting and explaining the evaluative discrepancy based on risk differentials in a way that shields the mere-difference view from that discrepancy necessitates abstracting from circumstances of social injustice. Barnes does not do that. Any attempt to employ the argument from risk differentials without references to social injustice must bear the burden of explaining why that differential can persist in the absence of social injustice. To do this in a way that does not compromise the mere-difference view would be contrived and implausible. Therefore, 
Barnes cannot show that the mere-difference view does not entail a specific stance on the cause/remove discrepancy.

\section{The Strategy of Denial: Denying the Discrepancy and Denying the Relevance of Common Sense}

The problem of the apparent evaluative discrepancy between Disabled Baby and Reverse Disabled Baby remains. The first argumentative strategy that was meant to defend the mere-difference view against that challenge - by accepting the discrepancy but explaining it with the resources of the mere-difference view - fails. Next up is the second potential response to the cause/remove discrepancy: denying it. According to Barnes, Cara acts wrongly both in Disabled Baby and Reverse Disabled Baby because in each case she interferes in the life of her child without justification. ${ }^{17}$ While the specific judgment on whether Cara acts rightly or wrongly is not entailed by the mere-difference view - but results from the noninterference principles that Barnes postulates - Barnes is correct to conclude that the mere-difference advocate should evaluate both cases as being on a par.

Coming to the same conclusion about causing a child's disability and causing a child's nondisability is counterintuitive and therefore a significant challenge to the mere-difference view. This is a challenge that Barnes is happy to meet. In a resourceful move, Barnes proclaims that the assessment of both cases being on a par is only counterintuitive if evaluated against the backdrop of commonsense intuitions. Indeed, this is so. If we remove the commonsense intuitions from the equation and either rely on mere-difference-view intuitions or no intuitions about disability at all, then the assessment that causing disability and causing nondisability are on a par is no longer counter to any available intuitions.

What are we to make of this argumentative strategy? Dialectically, the decision to simply discount commonsense intuitions about disability does not seem to be warranted. Recall that Barnes presents her paper as responding to the causation-based objection and that she develops detailed considerations to challenge specific parts of that objection. To declare thereafter that the very basis of the objection to be considered simply has no role to play in evaluating the relevant thought experiments may strike some as confusing. In essence, Barnes claims generally that the basis of any kind of similar argument - commonsense intuitions about disability - is unsound. Such a dialectical approach could only be justified if McMahan, Harris, Kahane or Singer had themselves forgone commonsense intuitions as the basis of their arguments. This is not the case.18 Nevertheless, Barnes seems to believe that the causation-based objection she discusses seeks to offer "independent traction" beyond the appeal to intuitions:

17 Ibid.: 103.

18 See, e.g., Harris (2001: 384); Kahane (2009, \10); Singer (2001: 133). 


\begin{abstract}
The causation-based objections are an attempt to do better - to get some independent traction on the mere-difference/bad-difference debate. They try to show that the mere-difference view has implausible, impermissible consequences, even by the lights of its defenders. In what follows, I argue that these causation-based objections do not succeed: they do not in fact give this sort of independent traction on the mere-difference/bad-difference debate. ${ }^{19}$
\end{abstract}

This assessment of the causation-based objections is false as their authors unanimously appear to rely on commonsense intuitions. Therefore, their arguments cannot offer traction independent of those intuitions. If those intuitions are discounted, their arguments are no longer convincing. The authors are aware of this. ${ }^{20}$ However, it is of course true that the authors of causation-based objections want to convince persons that the meredifference view has impermissible consequences. But who are those persons? As is commonly the case with these kinds of arguments, they are addressed at persons who have not fully considered all of the implications of their view and whose intuitions may conflict with those implications. Clearly, these arguments are not aimed at hardened defenders of a particular view who are - from the perspective of common sense - willing to simply bite the bullet. In summary, Barnes' critique of commonsense intuitions is at this point dialectically unconvincing.

On a more positive reading, however, one could interpret Barnes' decision to discount commonsense intuitions as the only defensible one for what good does it do to assess arguments that rely upon indefensible intuitions? According to Barnes, commonsense intuitions about disability are a bad guide to the wellbeing of people with disabilities because these intuitions are affected by ableist bias. That this is the case and that this gives warrant to discount commonsense intuitions about disability are the very basis of Barnes' paper. Without this assumption vindicated, her overall argumentative strategy fails outright. Unfortunately, she spends only one paragraph in defense of that assumption, basically citing the historical precedent of mistaken commonsense intuitions about minorities other than people with disabilities. ${ }^{21}$ Nevertheless, pointing to the potential fallibility of commonsense intuitions when evaluating the situation of minorities that have historically been discriminated against is one fundamental and laudable insight of Barnes' paper. Indeed, a dialogue between groups with solidified yet competing intuitions seems possible only on the basis of arguments that do not rely on such intuitions.

What remains to give traction if we relinquish normative intuitions about disability? Barnes suggests that in evaluating the mere-difference view we should not assume it to be "utterly unconstrained." Rather, "when its commitments are counterintuitive, it needs to be able to show how those commitments are nevertheless principled and consistent." ${ }_{22}$ If we interpret this position on the constraints of the mere-difference view

\footnotetext{
${ }^{19}$ Barnes (2014: 94).

${ }^{20}$ See the references in $\mathrm{n} .8$.

21 Barnes (2014: 104).

22 Ibid.
} 
as referring to principledness and internal consistency, it is certainly insufficient as an evaluative standard. According to this standard, all manner of fanatical views (religious, political and otherwise) would be acceptable. What - at a minimum - is required is that the view that is to be evaluated without reliance on normative intuitions about disability does not conflict with other basic intuitions and commitments that are not suggested to be affected by ableist bias.

\section{Independent Traction Revisited}

Before we can assess arguments that evaluate the mere-difference view without relying on normative intuitions about disability, we need to take a fine-grained look at the specific claims that the mere-difference view makes. As previously mentioned, proponents of the mere-difference view do not claim that disabled people are actually as well off as nondisabled people but only that they would be so in a just society. Therefore, the plausibility of the mere-difference view hinges on the definition of a just society. If one were to assume that a just society would bestow special privileges on disabled people (e.g., privileged access to positions of political, social or financial influence) it would make the mere-difference view more plausible than if one were to assume that a just society does not even have the duty to cover reasonable health care costs.

To emphasize the importance of this definition, consider that one could also simply define the just society as that society that makes it true on average that disabled people are de facto as well off as nondisabled people. On this definition, the mere-difference view would be true - but trivially so: The view would be vacuous.

To avoid such a vacuous view, let us assume - as Barnes implicitly appears to $\mathrm{do}^{23}$ - that a just society would simply not discriminate against disabled or nondisabled people and that they would on average experience similar social and socioeconomic circumstances. Also let us assume that while disabled and nondisabled people are offered reasonable health care in a just society, it is not the case that society must expend all or nearly all its resources on health care. With that out of the way let us consider a number of alternative arguments against the mere-difference view that in fact are meant and able to gain traction independent of baddifference-view intuitions and normative intuitions about disability.

One obvious difficulty of the mere-difference view relates to its scope. A number of disabilities often are accompanied by chronic pain and a reduced lifespan (paraplegia, tetraplegia, Down syndrome, mental disabilities, rheumatic diseases, asthma). Without relying on intuitions about disability, we can assess pain and early death as bad. Therefore, it is prima facie not plausible that the mere-difference view can apply to these disabilities.

Proponents of the mere-difference view can reply in three ways to defend the extensive scope of their view. First, they could claim that

23 Ibid.: 91-92. 
while indeed pain and early death are bad, these bads are outweighed by other goods that come with these specific disabilities. This would amount to the claim that these disabilities would actually be a good difference if the pain could be suppressed and early death could be avoided. Without relying on intuitions about disability, it is hard to counter this claim. However, it is worth pointing out how extreme that claim is. In a similar vein, proponents of the mere-difference view could point out goods that are conditional on pain and early death rather than conditional on having a specific disability. Examples of goods that may (in part) be conditional on pain or early death are the mental and emotional strength derived from pain and the resolve and sense of purpose derived from the anticipation of early death. However, these goods will, at least in part, be moral rather than prudential goods and thus not contribute to the overall wellbeing of a disabled person but rather make that person morally good. But even where the goods contribute to the wellbeing of a person, they seem to be unable to outweigh the bads - even when combined with other possible advantages that are conditional on having a disability. For example, it is implausible that the positive wellbeing effects of strength derived from pain outweigh the negative wellbeing effects caused by the pain necessary to gain strength. Otherwise, it would potentially be prudent to inflict pain upon oneself simply to gain strength. Disregarding highly particular circumstances this is implausible.

Second, proponents of the mere-difference view could claim that the commonsense intuitions that pain and early death are bad are simply misguided and that theories of wellbeing that support these judgments are wrong. This view has highly problematic implications and potentially gives rise to a number of inconsistencies. It contradicts some of the most widely shared moral intuitions and social practices. A defense of that view - if at all conceivable - would be very hard to substantiate. Therefore, the scope of the mere-difference view must be restricted to disabilities that neither cause (chronic) pain nor early death.

Third, advocates of the mere-difference view could attempt to abstract from pain and death and insist that their view is only about disabilities per se. This move, however, would render the mere-difference view practically irrelevant to the case of some significant disabilities. For, in real life, some significant disabilities cause pain and early death.

The scope of the view has to be further restricted with respect to the capacity of the relevant society to accommodate people with disabilities. Simply put, prior to the invention of the wheel, the mere-difference view was significantly less plausible with respect to certain mobility impairments. Similarly, today's technological advances in the health care sector strongly influence society's capacity to accommodate people with disabilities. If we assume that "ought" implies "can," this has a direct influence on the concept of the just society and therefore on the scope of the mere-difference view. The view is significantly more plausible with the backdrop of a highly advanced society. Accordingly, the scope of the view must be restricted to a certain era. In the same way - but motivated by considerations of cost-efficiency rather than the principle of "ought" implies "can" - the view is less plausible in the context of a society with 
comparatively many people with different kinds of disabilities that require different kinds of individually expensive treatments. People with diverse disabilities who require diverse kinds of assistance from nondisabled people are less likely to have a claim to that assistance the fewer nondisabled people there are. ${ }^{24}$

Another, if less obvious, difficulty of the mere-difference view pertains to its specificity. Defenders of the mere-difference view of disability deny not only the bad-difference but also the good-difference view of disability. Therefore, the mere-difference view is constrained on both sides of the spectrum of wellbeing and makes a rather precise claim about the differential effects of disabilities on wellbeing. Namely, on average, this effect will be about neutral in a just society - as neutral as the effects of sex, race or sexual orientation. ${ }^{25}$ From this claim two interrelated problems emerge.

First and simply put, little systematic differences in wellbeing levels between disabled people and nondisabled people are required for the view to fail. The strength of this criticism depends crucially on the effects of sex, race and sexual orientation on wellbeing. If these factors have a minimal effect on wellbeing, the mere-difference view claims that disabilities also have a minimal effect on wellbeing. The specificity of that claim alone makes the mere-difference view implausible. However, one may also argue - in opposition to the criticism proposed - that these factors cause substantial variances in wellbeing. For example, one may argue that women are substantially better off than men because, e.g., they live longer and are able to give birth. ${ }^{26}$ Let us assume that men are in fact substantially worse off even in a just society. Given this assumption, disability could accurately be described as a mere-difference even if people with a disability in a just society are substantially worse off than people without a disability. This result is troubling. If maleness makes people substantially worse off, one should deny that maleness is a mere-difference rather than claim that because disability is - in (at least) one important respect - like maleness, disability is a mere-difference as well. Alternatively, one could, of course, claim that maleness has worse effects on wellbeing than having a disability and that disability is, in fact, a mere-difference. These difficulties arise because Barnes understands "mere-difference" in relation to "features we standardly treat as mere-difference features." ${ }_{27}$ This approach is problematic because if we standardly but incorrectly treat fea-

24 Under different circumstances, the claim to assistance may even be strengthened by a relatively high number of people with a disability. Specifically, if economies of scale arise in the provision of assistance (as is the case in implementing accessible design), a person with a disability may be more readily entitled to that assistance because many other people with disabilities will benefit from it as well.

25 Barnes explains this at length in her (2009) "Disability, Minority, and Difference," Journal of Applied Philosophy 26: 337-55.

${ }^{26}$ Note that Robert Sparrow argues that "in some societies it is now - or soon will be better to be born a woman" (in his (2010) "Should Human Beings Have Sex? Sexual Dimorphism and Human Enhancement," The American Journal of Bioethics 10: 3-12; see also his (2012) "Human Enhancement and Sexual Dimorphism," Bioethics 26: 464-75).

${ }^{27}$ Barnes (2014: 94). 
tures as mere-difference, reliance on these incorrect judgments only perpetuates the problem. What is needed is a foundational understanding of mere-difference that directly refers to variance in wellbeing. Where precisely to draw the line between mere-difference and bad-difference is a challenging decision subject to vagueness. However, this should not distract from the fact that mere-difference must describe a narrow corridor if the view is supposed to have significance. Therefore, little systematic effects of disability on wellbeing suffice for such a view to fail. It is this specificity that makes the mere-difference view vulnerable.

A second and related problem that results from the specificity of the mere-difference view is that, according to the view, a great number of highly complex and phenomenologically highly diverse disabilities (blindness, deafness, paralysis, etc.) all have about the same effect on wellbeing in a just society - namely an about neutral one. Again, it is not impossible that the view is correct but it is not necessary to rely upon intuitions about disabilities to recognize that the view lacks plausibility. What would be needed is a theory that explains why such a diverse set of physical and mental impairments would yield such a predictable outcome in a just society.28 To appreciate just how far such an assessment is independent of intuitions about disability, consider whether it would be plausible that a great number of very different additional abilities would also be about neutral for wellbeing. To avoid the obvious critique that our intuitions about additional abilities are biased due to pop-culture phenomena such as Wonder Woman or Superman, consider extra abilities that you do not associate with these phenomena. The more diverse the set of abilities imagined, the less likely it will be that their effect on wellbeing can be about neutral - or neutral enough for them to warrant being called a meredifference. ${ }^{29}$ In fact, the same point can be made with reference to a large number of phenomenologically highly diverse experiences (e.g., being touched, being in the dark, feeling cold water, being isolated, feeling searing pain, hearing a bird sing). Even without reference to specific normative intuitions regarding the effect these experiences may have on our wellbeing, it is plausible to assess the likelihood that they all have the same effect on our wellbeing as minimal.

A third complication for the mere-difference view becomes apparent if we consider the effects of multiple disabilities in union. Jeff McMahan argues that if disabilities were truly neutral traits they would also have neutral effects on the wellbeing of a person who has multiple

${ }^{28}$ We discuss and critique one such view - that of David Wasserman and Adrienne Asch - below.

29 One might object to our argument that additional abilities might bring along positive as well as negative effects on wellbeing and that they are therefore mixed blessings - at least potentially. For this reason, one might further object that the overall effects of additional abilities might be neutral. However, even if we assume that additional abilities have both good and bad effects, this does not suggest in itself that their overall effect on wellbeing is about neutral. For either the positive effects might outweigh the negative effects or the negative effects might outweigh the positive ones. 
disabilities.30 According to McMahan, it may seem as if an individual disability were a neutral trait because a disability - such as blindness - may well be compensated for by heightened remaining abilities: e.g., hearing. However, if we contemplate the effects of multiple disabilities on an individual it becomes apparent that it is exceedingly unlikely that those effects will be neutral. To press his point, McMahan asks his readers to consider the case of an individual who has all disabilities and concludes that "it would be impossible to believe that that individual's life would not be worse than the lives of most others - or that it might be worse but only because of social discrimination and lack of adequate social accommodation." ${ }_{31}$ Strictly speaking, it is of course possible to believe that that individual's life would not be worse; however, it is deeply implausible that this belief is justified.

According to McMahan, the reason that this belief is unjustified is not that disabilities interact in special ways so as to have worse effects in union. Rather, he interprets the negative effects of disabilities simply as being additive. He does so based on an assessment of how one could best explain the negative effects that several disabilities would have on a person. In McMahan's view, the correct explanation largely relies on pointing out the negative effects each disability has individually as well as the fact that multiple disabilities make it more difficult to overcome the obstacles presented by each individual disability. According to this view, individual disabilities are - even if they do not actually reduce the overall wellbeing in each case - obstacles to wellbeing that need to be overcome. If it is warranted to assume that individual disabilities are in fact obstacles to wellbeing - even if they are not necessarily insurmountable - it becomes clear that the bad-difference view is plausible.

While McMahan's argument is intuitively plausible, it does not stand unopposed. David Wasserman and Adrienne Asch have recently objected to it. According to them, the argument rests on a false assumption - that one cannot live as well without as with any given ability unless compensation for the absence of that ability is available - and fails because, while people with disabilities may have fewer ways to realize important goods, the ways available to them may be as effective as the ways they are precluded from. As Wasserman and Asch put it, "Having more ways to realize a good does not mean that you can realize it more fully." ${ }_{32}$ In a nutshell, their view is one of "saturation." ${ }_{33}$ They assume that "a blind person can live as well as a sighted one not because she develops better hearing - she may not - but just because the senses and abilities she has are more than adequate to allow her to live as fully and richly as possible." ${ }_{34}$ It bears noting that, according to this view, a person with dis-

${ }^{30}$ McMahan (2005: 96).

31 Ibid.

32 D. Wasserman and A. Asch (2014) "Understanding the Relationship Between Disability and Well-Being," in J. E. Bickenbach, ed., Disability and the Good Human Life, New York: Cambridge University Press, pp. 139-67.

33 Ibid.: 157.

${ }^{34}$ Ibid. 
abilities cannot only live a life worth living, or a life that is sufficiently good, or a life that is as good as the average life of a nondisabled person, but a life that could not be fuller or richer. Simply put, additional abilities are not needed because they do not enable their bearer to live a better life.

Let us assume for the sake of argument that Wasserman and Asch's conception of wellbeing is essentially correct: You can flourish to the fullest extent as long as you have access to any effective ways to realize important goods. This assumption explains why it would be very bad for you to suffer from all (or sufficiently many) disabilities while at the same time it seems that individual disabilities are not bad for you as long as alternative ways to realize important goods are available to you. Even though, given this assumption, McMahan's argument cannot be maintained in its original form, a negative assessment of each individual disability remains justified: Each individual disability precludes a way to achieve important goods and, given that the precluded way may be one of the only effective ways for you to achieve an important good, each disability is associated with a certain risk of being bad for you. Moreover, given this risk, you are prudentially advised and morally required to avoid causing disabilities. Thus, even given Wasserman and Asch's theory of wellbeing, McMahan's argument speaks in favor of something like the bad-difference view.

A further problem with the view of Wasserman and Asch exists: Notice that, on their conception of wellbeing, important goods need to be understood in a rather coarse-grained way. Obviously, the blind person cannot take pleasure in visual aesthetic experiences; the paraplegic person cannot accomplish a marathon run; the mute person cannot sing in a choir. If these activities and experiences - the descriptions of which are rather fine-grained in that they involve seeing, running and singing - were themselves important goods and not just ways of achieving important goods, then the objection put forward by Wasserman and Asch would fail. What they want to emphasize is that there are ways for blind people to have aesthetic experiences, for paraplegic people to accomplish athletic goals and for mute people to take part in collective achievements, and that these ways are sufficient to flourish to the fullest extent. Wasserman and Asch thus must assume a coarse-grained conception of important goods.

This assumption is problematic because if we accept a coarsegrained conception of important goods as Wasserman and Asch do, then the ways to achieve these goods - even if they may not be important goods themselves - seem to matter in terms of wellbeing. One person may enjoy visual aesthetic experiences but be indifferent toward music, another person may be a devoted runner but not care at all about other sports and so on. Missing out on specific ways to achieve coarse-grained human goods, it seems, can itself make you worse off. ${ }^{35}$ Thus, the availa-

35 Notice that this observation is completely independent of intuitions about the impact of disabilities on wellbeing. 
bility of each individual way to achieve important goods may itself be good for you, even if alternative effective ways to achieve these very important goods are open to you. Of course, you may be lucky in that a given disability might not preclude the single best-for-you way to achieve an important human good. On the other hand, maybe it does. This provides a second reason, associated with risk, that one should avoid causing disabilities. 36

In objection to this critique, one may argue that (i) a person born with a disability lacks the basis for valuing and is therefore unlikely to come to value the specific ability she is precluded from and that (ii) if she were to come to value this ability, this valuing would be no more illadvised - and no more harmful - than a person without a disability coming to value high-level, yet unachievable, functioning of any kind. Regarding (i), it bears noting that the challenge has some plausibility in the context of disabilities related to sensory experience but cannot be applied to disabilities related to motor functioning. A person born without limb function may very well - and quite independently of any unjustified interferences from society - develop the strong wish to walk. Of course, this preference is not developed by all those born without limb function but persons born without limb function neither lack the basis for valuing limb function nor is it unlikely that they do value it. However, even with respect to sensory experiences it is not clear that persons who lack a particular ability such as seeing would lack the basis for developing a preference for that ability. Consider, for example, the blind person who develops a preference for seeing based on the reports of her friend regarding the advantages of being able to see.

With respect to (ii), it bears noting that while maladaptive preferences and unfulfillable aspirations are always problematic, there are good reasons to believe that they are considerably more problematic in the case of disabilities rather than non-exceptional abilities. Not being able to play basketball in the NBA may be rough for those who dreamed of doing so all along. However, not being able to play in the NBA is fully compatible with a high level of basketball talent. Being able to function at a particularly high level cannot be compared in importance to functioning at all in a particular domain.

However, all that neglects the basic question: Would people with a disability be better off in the counterfactual scenario in which they do not have that disability? That could be true quite independently of whether they develop a preference for overcoming their disability. Of course, it is

\footnotetext{
36 Maybe Wasserman and Asch would object that their conception of wellbeing is incompatible with the assumption that the ways to achieve important goods themselves matter in terms of wellbeing because, on this assumption, (i) access to important goods does not guarantee flourishing to the fullest degree and maybe also because (ii) ways of achieving important goods would themselves qualify as goods (albeit not important goods). If the view of Wasserman and Asch were indeed incompatible with the assumption that the ways to achieve important goods matter themselves in terms of wellbeing, this would provide an argument against their view, and thus undermine their objection to McMahan's original argument, because the mentioned assumption is plausible.
} 
not true in all cases that persons are better off if they can, e.g., see; however, we claim that this will typically be the case.

\section{Conclusion}

Barnes reveals an important challenge for proponents of the baddifference view: Some defenders of the mere-difference view are prepared to simply accept the counterintuitive implications of their position. From the perspective of such hardened defenders of the mere-difference view, any criticism on grounds of commonsense intuitions will run the risk of being judged a mere expression of ableist prejudice. Thus, a dialogue between such proponents of the mere-difference view on the one side and adherents of the bad-difference view on the other requires arguments with independent traction. We have presented several such arguments to the effect that the mere-difference view needs to be significantly reduced in scope - and may turn out to be false altogether. ${ }^{37}$

Vuko Andrić

University of Mannheim

Research and Study Centre "Dynamics of Change"

vandric@mail.uni-mannheim.de

Joachim Wündisch

Bielefeld University

Center for Interdisciplinary Research

and

Heinrich Heine University Düsseldorf

Department of Philosophy

joachim.wuendisch@uni-duesseldorf.de

37 The authors would like to thank Matthew Baum, Dieter Birnbacher, Frank Dietrich, Andrea Klonschinski, Stefan Riedener, Julian Savulescu, Holmer Steinfath, audiences at the universities of Düsseldorf, Göttingen and Oxford, as well as the editors and anonymous reviewers of the Journal of Ethics and Social Philosophy, for their helpful comments. 